\title{
High Resolution Electron Microscopy of CdSSe Nanowires
}

James K. Walton, Sergei Rouvimov, and Raj Solanki

Department of Physics, Portland State University, Portland, OR 97207-0751

The band gap of the ternary alloy CdSSe can be tuned from about $2.44 \mathrm{eV}$ (for CdS) to $1.72 \mathrm{eV}$ (for $\mathrm{CdSe}$ ) to cover almost the entire visible spectrum, hence is of particular interest for fabrication of optoelectronic devices. Growth of planar epitaxial thin films of this alloy requires a substrate with a close lattice match. This requirement has been a major obstacle for fabricating semiconductor-based optoelectronic devices (such as lasers, LEDs, and detectors) with controllable and widely variable operating wavelengths. However, this limitation can be overcome by growing single crystal CdSSe nanowires with variable composition (hence the bandgap) that can be grown on a wide range of substrates, including amorphous substrates [1-3]. We have achieved growth of CdSSe nanowires over a wide composition range and have examined their bandgaps and morphologies of these 1D structures using photoluminescence, energy dispersive x-ray spectrum (EDS) with a scanning electron microscopy, and high-resolution transmitted electron microscopy (HRTEM, FEI Titan). The nanowires were grown in a horizontal furnace with a $5 \mathrm{~cm}$ diameter quartz tube that acted as the deposition reactor. The deposition was achieved at low pressures (10 mTorr) and the sources were CdSe and CdS powders. The composition of the nanowires was varied by adjusting the molar ratio $(\mathrm{CdS} / \mathrm{CdSe})$ of the source powder. Typical source and substrate temperatures were $730{ }^{\circ} \mathrm{C}$ and $650{ }^{\circ} \mathrm{C}$ and the growth time was $15 \mathrm{~min}$. The vapor was transported to growth region of the reactor with an Ar flow of $50 \mathrm{sccm}$. The substrates were composed of silicon wafers with a $1 \mu \mathrm{m}$ thick layer of silicon dioxide that was coated with a $4.5 \mathrm{~nm}$ thick layer of gold film. The gold film allowed us to employ vapor-liquid-solid (VLS) mechanism to grow the nanowires [4].

A side view of the as-grown nanowires observed with a scanning electron microscope is shown in Fig.1. The diameter of these nanowires ranged from about $20 \mathrm{~nm}$ to $80 \mathrm{~nm}$ and their length was typically tens of microns. The nanowirres have been studied by HRTEM either at a FEI Tecnai G2 F20 ST electron microscope (at Portland State University) or at an aberration-corrected FEI Titan 80-300 (at CAMCOR of the University of Oregon). The majority of NWs are monocrystals of wurtzite structure with the growth direction of (Fig. 2). The range of composition of the grown nanowires is plotted along with Vegard's Law (solid line) in Fig.3.

\section{References:}

1. X. Duan et. al., Nature 421 (2002) 24

2. R. B. Liu et. Al., J. Phys.: Condens. Matt. 19 (2007)136206

3. A. L. Pan, et.al., Nano Lett. 9 (2009) 784

4. R. S. Wagner and W. C. Ellis, Appl. Phys. Lett. 4 (1964) 89 


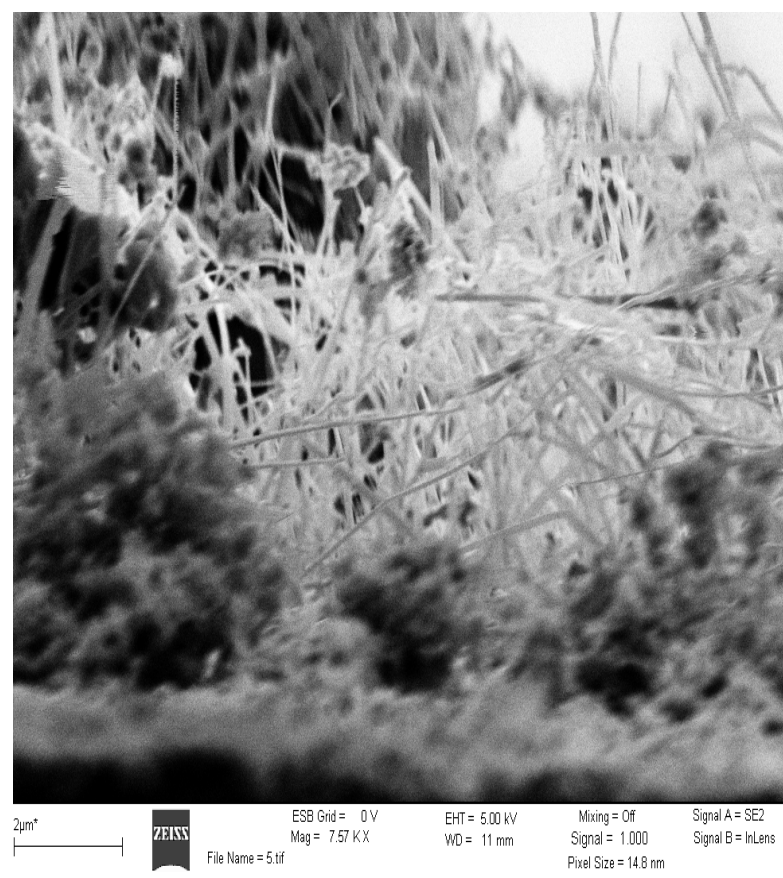

FIG 1. SEM side view of the CdSSe nanowires. The scale marker is $2 \mu \mathrm{m}$ long.

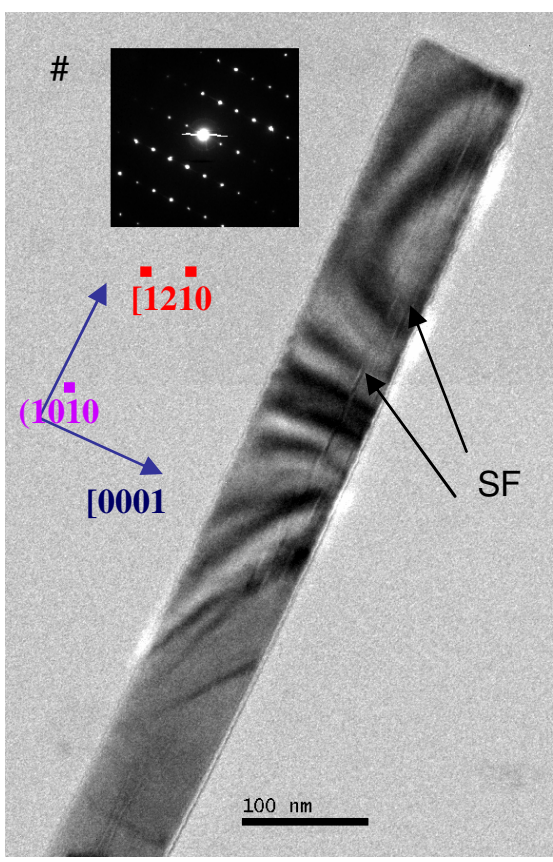

FIG 2. TEM image of CdS. Inserted is SAED.

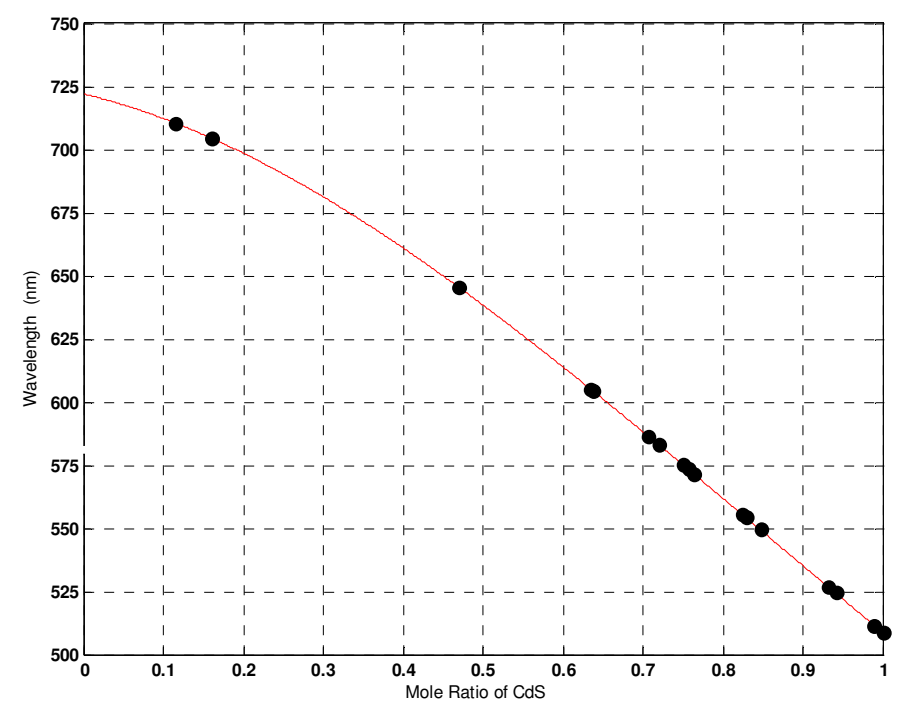

FIG. 3. Bandgap (in terms of emission wavelength) versus the mole ratio of CdS. The solid line is Vegard's Law. 